\title{
The Digital Electronic Media Methods in Communication with the Public: BBC Website as a Model
}

\author{
Dr. Hashim Alhamami \\ Press and Media Department, Arab Open University \\ P.O. Box 1339Amman. Postal code 11953, Jordan \\ Tel: 00962-786583823,E-mail: dr.hashim69@gmail.com
}

\begin{abstract}
The study aimed to explain most of the digital electronic media methods in communication with the public on the BBC Arabic website. To achieve the objectives of the research, the qualitative descriptive method was used by content analysis and observation. The results of the research found that the design of the BBC Arabic site is easy to use because of the logic of organizing the content. There is easy access to information and news on the site. The Multimedia is one of the most important additions to the website, especially the news sites. The most important multimedia used in news sites is the process of integrating text, graphics, sound, image, links, and tools that allow the user to interact and communicate. Moreover, the BBC website contains a diversifying the content by providing multimedia that includes text, still images, video, audio, descriptive features of all images, audio and video clips. As well as, the BBC Arabic site features a distinctive style in its multimedia display by using playable files with more than one player such as Real Player, Player or Windows Media. Also, the news sites are interested in providing a variety of information materials: texts, audio clips, videos, photo albums and more. In addition to several copies of their sites in languages other than Arabic. Finally, the interactive services on the BBC News website help attract users and build a trust-based relationship between the site and its audience. The research recommended creating the appropriate climate, adequate resources, and appropriate cooperation to improve the digital electronic media methods across the Arab world as a primary for communication with the public
\end{abstract}

Keywords: $B B C$ News Website, BBC Arabic, Digital Electronic Media, Multimedia

DOI: $10.7176 / \mathrm{NMMC} / 81-08$

Publication date:June $30^{\text {th }} 2019$

\section{Introduction}

Nowadays electronic media represented as an interchange of three paths of technological evolution. The first refers to the improvement of technologies bridging space. The second refers to the development of technologies for bridging time. The finally line turn on from making computations by moving limestone scree on board (the origin of the verb calculate), utilizing the abacus and mechanical calculators to the digital computer. The final interchange of these three lines to digital Information and Communication Technology (ICT) formed a new form of media utilize to communicate, describe by the potential to transfer information at almost zero cost by space and time. (Coroama et al., 2015).

Moreover, interchange explained a key analytical idea in realization the reinvention of media organizations as multimedia. Since the 1980s, this idea of interchange has been associated with the key part that technology has always played in media growth, an influence concerning to digitalization and the World Wide Web in the last few decades. Digitalization has pliable media content to multiply and flow faster, minimizing the importance of the transmission source and medium. Further, Digital-based systems have also faster the internal journalistic operation for content sharing and creation - collecting, archiving and editing. For its part, the development of the web in the middle 1990s represented a new media platform with further benefits for content distribution - cost-effectiveness, ubiquity, constant updating, connecting, multiple media gathering, unlimited publishing space and interactivity (Larrondo, 2014).

From other and, the media is part of the communication process, an activity that is achieved to achieve certain goals and uses the means of mass communication. Knowledge to individuals and groups in order to influence their minds, feelings, and activities. The Internet is a new communication medium for the media. It is a hybrid communications technology that has more than one communication style: personal, collective and public, with no clear boundaries separating these forms. The network is one of the most popular uses of the Internet and uses the network news transfer protocol (NNTP), which regulates the way the distribution of news articles and retrieved 
and sent and inquire about. Moreover, the network offers bulletin boards and Chatting Rooms, and the Net News network consists of a huge system that includes a large number of open and ongoing dialogue forums called News Groups. These groups continue to work around the clock and throughout the year, Browsers allow access to newsgroups where a user can follow an open dialogue without being involved. This is called Lurking, which encourages visitors to newsgroups to enter the conversation and send an article to the group of their choice Senet is very fast. Group managers determine how long messages are posted before the system cancels them. Groups do not keep their messages published for more than a week. Electronic media is provided in a single format (text, image, video sound ...) or multimedia. Websites often offer services designed to enhance communication and interaction with the recipient (McQuail, 2010).

Besides, the BBC's Internet site was the first multimedia news site where multimedia ads were posted on the site with great capabilities utilizing audio and video technologies. In 2003, BBC Arabic took an important step in transferring information to Arab recipients through The use of multimedia that pool audio and image stimulates the audience to participate effectively and instantly. Of course, multimedia is now the feature of the majority of news sites that have benefited from its advantages to transmit image, sound, and speech at the same time (Quinn, 2005). Thus, this research came to explain most of the digital electronic media methods in communication with the public on the $\mathrm{BBC}$ website.

The importance of this research comes from both scholarly and practical contributions to the field of media, particularly the new research agenda that is electronic media. The primary scholarly contribution is through facilitating a greater understanding of the digital electronic media methods in communication with the public. Further, this understanding is facilitated within the multimedia that uses to communicate with the public on the $\mathrm{BBC}$ website. Also through understanding and explain the methods of digital electronic media to communicate with the public on the $\mathrm{BBC}$ website. Finally through understanding usefulness of electronic media of communication with the public in $\mathrm{BBC}$ website.

Also, this research has practical significance. Specifically, electronic media have to increase build better relationships with their existing readers and listeners. Moreover, electronic Media face high effectiveness, ubiquity, constant updating, connecting, and multiple media gathering, unlimited publishing space, and interactivity, and thus electronic Media would be interested in understanding how they can cultivate readers and listeners trust, and promoting them online participation.

As well as, research indicates that new media such as using multimedia at electronic media have the potential to bring more reader, for instance, they can attract more readers from different ages. Multimedia, which can allow electronic media to deal with readers and listeners more intimately. There are many types of multimedia; however, this research focuses on digital electronic media methods in communication with the public.

Nowadays readers and listeners maybe not interact with traditional media dimension, but actively engage with electronic media according to its role on interchange explained a key analytical idea in realization the reinvention of media organizations. Thus, this research aimed to explain most of the digital electronic media methods in communicate with the public. So, this paper will try to answer the following question:

- How is the ease of use digital electronic media to communicate with the public on the BBC website?

- What is the multimedia that uses to communicate with the public on the BBC website?

- What is the methods of digital electronic media to communicate with the public on the BBC website?

\section{Objectives of the Paper}

The main objectives of the paper are:

- To explain the ease of use the digital electronic media of communicate with the public on the BBC website.

- To explain the multimedia that uses to communicate with the public on the BBC website.

- To explain the methods of digital electronic media to communicate with the public on the BBC website.

\section{Theoretical Framework and Previous Studies}

Media is a sustainable source of shared images and messages. Moreover, Technology of media is an important part of a person's lives in the twenty-first century and play a very important role in creating awareness related to various aspects of life and personality. Add to that, Electronic media stimulate and support attitude and contribute significantly to the formation of modern attitudes. On other hand, Media have performed an important part in the 
moral improvement of youth (David-Ferdon, \& Hertz, 2007).

The history of electronic media returns to the invention of radio and to the first radio terminal, which was established in Pittsburg, New York, and Chicago at 1920s. Next, to the USA, European countries begin started radio stations for broadcasting news and entertainment content. Moreover, the next phase in electronic media history returns to the contrivance of cinema. After that, television broadcasting began in the US on an experimental basis during the 1920s. However, the dramatic effect of television as a medium began in the 1950s. Equivalent to these, the recording industry also flourishes in the western countries. In short, the term electronic media mainly include Radio, Television, and Audio and Video records (Meyrowitz, 1986).

Furthermore, the beginning of the electronic press was the result of cooperation between the BBC and the Independent Broadcasting Corporation (IBA) in 1976 as part of the Teltext service. The first enterprise system appeared under the name Ceefax, while the second enterprise system was known as Oracle. In 1979, a second, more interactive service was introduced in Britain, known as the TEXT video service with the Prestel system provided by the British Telephone Association (BTA). Although the attempts of these institutions did not achieve the required success, but it changed completely at the beginning of the nineties, which brought with him tremendous developments at all levels, and if the success of the service of Tele Text due to reliance on the television, the success of the newspaper is directly linked to the availability of computers and evolution Programs that facilitate access to the Internet and deal with it. In the 1990s, the Internet has played a prominent role in the dissemination of information materials in various forms and forms in many languages and benefited many media, especially newspapers, which increased the number of their sites on the Internet significantly during the nineties. In 1992, Chicago Online created the first online newspaper on America Online and the first Internet online news site, launched in 1993 at the University of Florida's School of Journalism and Public Communication, Palo Alto, followed by another site on 19 January 1994, Alto Palo Weekly The first newspaper to be published on a regular basis. The newspaper is the first model to enter the electronic press industry in a large and increasing manner, especially with the provision of free Internet in the United States and the developed world, so that the press has become part of the development and distribution of the Internet. The majority of American newspapers began publishing online in 1994-1995, and the number of American daily newspapers that created websites increased from 60 in late 1994 to 115 in 1995 and 368 in mid-1996 (Malone, Yates, \& Benjamin, 1994: Inglis, Joosten, \& Ling, 2003: Gurevitch, Bennett, Curran \& Woollacott, 1982).

Moreover, the Washington Post is the first American newspaper to carry out a project worth tens of millions of dollars, including a paper prepared by the newspaper every time events, change with documentary references and classified ads. The project was called "paper ink" The new newspapers are the first of its kind in the history of paper, inks and in the traditional system of editing and reading, to use the computer and its vast possibilities in distribution across continents and countries without barriers or restrictions. This pioneering project was only in response to the rapid developments in linking technology And the emergence of multi-media systems, and verified the sleep of the Internet vertically and horizontally and the breadth of users and subscribers in the United States and many other countries, especially in the West, and start before establishing special sites for information, including specialized news information such as Sports, science and so on (Malone et al., 1994: Inglis et al., 2003 : Gurevitch et al., 1982).

In 1991, there were only 10 newspapers on the Internet, and this number increased to 1,600 in 1996 . The number of newspapers in 2000 was 4,000 on the Internet. Around $99 \%$ of the large and medium-sized newspapers in the United States have placed their pages on the Internet. As for the construction of the news content of the Internet press has evolved through three stages; in the first phase was the Internet newspaper re-published most or all or part of the content of the mother newspaper and this type of journalism is still prevalent. The second stage is to reproduce some texts to conform to the features of the network, by feeding the text with links, bookmarks, etc. This represents an advanced level of the first type. In the third stage, the journalists produce content for the Internet newspaper, in which they collect the publishing organizations and apply new forms to express the news (Inglis et al., 2003)

As well as, electronic media defined as media that use electronics power for the end user (audience) to arrival the content. This is in contrast to static media (mainly print media. The primary electronic media exporter familiar to the general public video recordings, audio recordings, multimedia presentations, slide presentations, CD-ROM and online content. Generality new media are in the form of digital media. Further, electronic media may be in all digital electronic data format. Any tools used in the electronic communication process (e.g. television, radio, telephone, desktop computer, game console, handheld devices) may also be behold electronic media (Chinwendu , 2015). 
Correspondingly, media can be outlined as technologies that intended to storage and dole out meanings. Generally, through media, mass media can be select out concerning the meanings created and the public receiving them. Concerning the meanings, mass media contents are produced by specialized agencies according to a predetermined agenda of "(daily) actuality" within the community (Wimmer and Dominick, 2013).

Furthermore, the mass media tools aimed at those with media technology products. With an obvious explanation, communication is executed by the press, cinema, film, radio, and television. With these tools, a message from one place (individuals, organizations, communities) is conveyed to a wide public in the operation or activity falls within the scope of mass communication. Moreover, Mass communication is a completely modern phenomenon of civilization. Mass media has helped not only with the socialization of the individuals but also to the community because its assistance in human improvement is necessary and inevitable. The socialization contact size occurs through the mass media. Particularly, mass media is closely adopting to transfer the data from the central area of the nation to the rest of the population (Öznacar and Dagli, 2016).

It was found through the reviewing of previous studies and researches - within the limits of the researcher's knowledge - that the digital electronic media methods in communication with the public on the BBC website have not been studied in its concept of the current research. Roth (2010) study aimed to reveal the scientific content contained in the articles published in the electronic media, using the curriculum content analysis of a sample of articles in the British BBC site http://www.bbc.co.uk/news. The findings indicated that the published scientific articles focus on several scientific fields such as natural sciences, humanities, and applied sciences. She also pointed out that the scientific articles published on the electronic media focus on a number of terms such as mind and culture, while the study found that electronic media focus more on some human sciences such as the individual's role in society, his place and his involvement in community interactions.

Add to that, Handayani, (2012) study aimed to explain to how media convergence influences Agenda-setting, Cultivation and Critical Theory and Culture Study Conception. moreover the study aimed to explained the effect of Mass communication, which has created media effect theory and cultural study as well as critical theory during its development, cannot be separated from its massive extend manner towards the public that could influence individuals, community, and cultural value. The result showed that media convergence has critically reduced mass communication features. Also the result represented that theory of agenda setting and cultivation have been revisited and shown that sources (media institution) and message play major role in old media but not in the new media. Agenda setting theory in new media shifted to the extent of what issues people tell the media they want to think about

Similarly, Chinwendu (2015) study aimed to explain Electronic media as an instrument has established as modern options for enhancing mass media. Further, as the number of technologies of electronic media in community increases rapidly, so does the rate of misuse. Even the most experienced members may be susceptible to unintentional mistakes. Maintaining professional boundaries in all forms of communication, technology-related or not, is vital to maintaining the public trust and appropriate professional relationships with students. Members must be aware of the many challenges and the ramifications associated with the use of electronic media. Moreover, Electronic media is now used formally in primary, secondary and tertiary institutions and informally in Nigeria. Moreover, adopt comes with many benefit and disadvantages; incorruptibility is therefore the key to insure that they are not used inappropriately. In formal learning, electronic media technologies should be highly maintained to ensure continuity and teachers trained on its usage as well as students. Informally, radio and television stations in Nigeria should develop more time to educational programmers. Media can be outlined as technologies that intended to storage and dole out meanings. Generally, through media, mass media can be select out concerning the meanings created and the public receiving them. Concerning the meanings, mass media contents are produced by specialized agencies according to a pre-determined agenda of “(daily) actuality” within community.

\section{Methodology of the research Paper}

The qualitative methods were used in this research; The descriptive approach is characterized by several advantages, the most important of which are: It studies the reality of the phenomenon and describes its characteristics accurately, and expressed qualitatively and quantitatively; so that researchers can know the extent of the phenomenon and its degree of association with the variables of the study, and then reach conclusions that help understanding Reality and development, as the descriptive approach is a scientific method used in the preparation of scientific research, specifically in the study of social phenomena and humanity (Creswell, 2012).

As well as, the descriptive approach is a comparative study of the variables in the study, its evaluation and interpretation in order to reach meaningful generalizations that increase the knowledge of the subject of the study. 
This method is one of the most common and used methods in the field of human sciences. The descriptive approach is defined as: Based on sufficient and accurate information on a particular subject or phenomenon in a known period and time periods, to obtain practical results and then analyze and interpret them in a manner consistent with the actual data of the phenomenon (Creswell and Clark, 2011).

\section{Sources of information}

Two main sources of information were used:

- Primary sources: The sources through which the researcher obtained the information by addressing the analytical aspects of the research, so that the primary data for the research was collected through: the site of the $\mathrm{BBC}$ and the content analysis of the site; how the site use multi-media on-site to interact with and communicate with the public, and the observation.

- Secondary sources: through the review of books and periodicals and references, Arab and foreign related, and research, articles and studies that dealt with the subject of research.

\section{Research population and sample:}

The research population was limited to the BBC website as a research model (http://www.bbc.com/arabic). The research sample consisted of the $\mathrm{BBC}$ website, which was chosen as a descriptive sample for the subject of the research, and all the materials presented were analysed through multimedia provided by the BBC.

\section{Limits of spatial and temporal research:}

The researcher studied the BBC website. During the period from 01/2/2019 to 31/3/2019 as an elected period to obtain the required information to achieve the objectives of this paper.

\section{Discussion and Findings}

This part of the research presents the findings of the research through the use of direct observation and tracking of the content of the BBC site.

\section{- Evaluation of the general appearance of BBC site}

Table (1) presents the results of the basic evaluation elements of the general appearance of BBC site: Headlines and colors, size and types Fonts, Consistency of patterns and colors, Background and Page consistency

Table (1): The Basic Evaluation Elements of the General Appearance of BBC Site

\begin{tabular}{|c|c|}
\hline Content analysis & BBC \\
\hline Headlines and colors & $\begin{array}{l}\text { 1- Use Easy addresses. } \\
\text { 2- Clear and prominent address, using dark blue color. Styles } \\
\text { and colors are consistent and comfortable to look within } \\
\text { the background. } \\
\text { 3- Using Dark gray within a white background inside pages. } \\
\text { 4- The address connected with link to another page. }\end{array}$ \\
\hline Size and types fonts & $\begin{array}{l}\text { 1- } \text { Clear and large line for comfortable reading } \\
\text { 2- } \text { Font size in titles } 19.5 \text { (Arial bold). } \\
\text { 3- } \text { subheadings font (Arial 11) } \\
\text { 4- text font Times New Roman (9.5) }\end{array}$ \\
\hline Consistency of patterns and colors & $\begin{array}{l}\text { 1- Consistency colors and patterns in titles And texts at all } \\
\text { pages of the site }\end{array}$ \\
\hline Background & 1- The site depends on white color backgrounds \\
\hline Page consistency & $\begin{array}{l}\text { 1- All pages are consistent in the site and follow the same } \\
\text { format and design. }\end{array}$ \\
\hline
\end{tabular}

From the above table the overall appearance of the BBC Arabic site shows that it is committed to the standards of ability to use. The titles are clear and prominent in clear and distinctive color and clear text. Moreover, the patterns and colors are consistent and comfortable. The font size is relatively small but the lines in all media materials. 
Also, the font size is relatively small; however, the lines in all materials were well visible and did not require any effort to read.

\section{- Web site efficiency}

Table (2) presents the results of the criteria related to web site efficiency to BBC site: Home page, Average download time, Size of image files per page, Logo, and Possibility return to home page.

Table (2): The Criteria Related to Web Site Efficiency to BBC Site

\begin{tabular}{|c|c|}
\hline Content analysis & BBC \\
\hline Home page & $\begin{array}{l}\text { 2- Includes different sections can be used to identify all the } \\
\text { news on the Middle East and the world. } \\
\text { 3- Contain a set of services and products. } \\
\text { 4- Access to mobile phone copy service. } \\
\text { 5- Weather news, live broadcasts of BBC television and } \\
\text { radio, information on the site and conditions for its use. } \\
\text { 6- All titles and images contain a links. }\end{array}$ \\
\hline Average download time & 1- $\quad$ Bites 1.494 .419 \\
\hline Size of image files per page & $\begin{array}{ll}1- & 171 * 304 \\
2- & 81 * 144\end{array}$ \\
\hline Logo & $\begin{array}{l}\text { 1- The logo of the site at the top of the page and clearly } \\
\text { 2- The home page translates to English version of which } \\
\text { page it was. }\end{array}$ \\
\hline Possibility return to home page & $\begin{array}{l}\text { 1- Possibility to return to the home page through notification } \\
\text { at all pages. }\end{array}$ \\
\hline
\end{tabular}

From the above table the site's proficiency results show that the commitment of the BBC Arabic website to the required standards. The Logo is clearly displayed in the upper right corner of the page, maintaining the same size on all pages. Similarly, the main page of the site contains different sections where each can be identified what is published on the pages of the site of the most important Arab and international news, in addition to the news of the Middle East and the world and the latest developments, and a range of services and products, including weather news, live broadcasts of the BBC TV and Radio, access to mobile phone service. Furthermore, the homepage of the site gives the user an overview of all the available pages on the internal pages. It also places the possibility of access and utilization of all its services, which highlights its efficiency and importance to the user.

\section{- Organize media information}

Table (3) presents the results of Organize media information in BBC site; Methods of, displaying contents of site, Icons, and Technical structure. Moreover, usefulness use and browsing in BBC site; the clarity, simplicity, and consistency of the browsing throughout the site Browsing tools and power. 
Table (3): The Organize Media Information in BBC Site

\begin{tabular}{|c|c|}
\hline Content analysis & BBC \\
\hline Methods of displaying contents of site & $\begin{array}{l}\text { 1- The design distinguished due to the logical organization } \\
\text { of the content. } \\
\text { 2- Easy access to information within the site. } \\
\text { 3- High flexibility in text handling, easy access to user- } \\
\text { friendly information }\end{array}$ \\
\hline Icons & 4- Contain menu and sub menu \\
\hline Technical structure & 1- Site port by Java script Which provides flexibility to users \\
\hline $\begin{array}{l}\text { The clarity, simplicity and consistency of } \\
\text { the browsing throughout the site }\end{array}$ & $\begin{array}{ll}\text { 1- } & \text { Easy browsing. } \\
\text { 2- } & \text { Retrieval information speedy. } \\
\text { 3- } & \text { Ease to dealing with site }\end{array}$ \\
\hline Browsing tools and power & $\begin{array}{l}\text { 1- } \text { Clearly browsing tools. } \\
\text { 2- } \quad \text { Flexible and efficient in use. }\end{array}$ \\
\hline
\end{tabular}

From the above table the design of the BBC Arabic site is easy to use because of the logic of organizing the content. There is easy access to information and news on the site. All the paragraphs are on the main page. The browser does not have to enter the internal pages unless the viewer want to read articles published in previous days. The site is a portal with several sections of news and reports, weather news, links to social networking sites, and other services. The site also provides abundant information and flexibility in dealing with the text and easy to get what suits the user information directly. Furthermore, each media item is accompanied by relevant links, related topics and more news, and from other news sites where the BBC Arabic website provides links to other news sites to convey different views of the story. In addition to the pictures, which are accompanied by most media materials, the BBC Arabic site provides a picture of the most important events. Besides, BBC Web site uses the animated newscast and uses the Java program which allows for speed, flexibility, and ease of use, in addition to adding an aesthetic element to the site.

\section{- Multimedia}

Table (4) presents the results about multimedia that uses in BBC site; Images Video and audio, and Adopt caption in images, Video and audio.

Table (4): The Results Multimedia that Uses in BBC Site

\begin{tabular}{|c|c|}
\hline Content analysis & BBC \\
\hline Images & $\begin{array}{l}\text { 1- The site provides pictures for important events and } \\
\text { provides detailed information about the image such } \\
\text { as description, comments and the date of the } \\
\text { photograph. }\end{array}$ \\
\hline Video and audio & $\begin{array}{l}\text { 1- A distinctive style for viewing video and audio files } \\
\text { 2- Speed in loading } \\
\text { 3- possibility of viewing file in more than one program }\end{array}$ \\
\hline $\begin{array}{l}\text { Adopt caption in images, Video and } \\
\text { audio }\end{array}$ & $\begin{array}{l}\text { 1- provides detailed information about Adopt caption in } \\
\text { images, Video and audio }\end{array}$ \\
\hline
\end{tabular}

Multimedia is one of the most important additions to the website, especially the news sites. The most important multimedia used in news sites is the process of integrating text, graphics, sound, image, links, and tools that allow the user to interact and communicate.

From the above table, and after viewing the BBC website, it was noted that the site is diversifying the content by providing multimedia that includes text, still images, video, audio, descriptive features of all images, audio and 
video clips. The BBC Arabic site features a distinctive style in its multimedia display by using playable files with more than one player such as Real Player, Player or Windows Media.

\section{- Diversify of content options}

Table (5) presents the results about Diversify of content options; Audio content, Video , search engines, Languages, Site Customize ,The methods that used in thematic classification of the media content , The methods used in the geographical classification of the media content, Methods of qualitative classification of media content, and Interactive Services

Table (5): the Content submission methods

\begin{tabular}{|c|c|}
\hline Content analysis & BBC \\
\hline Audio content & 1- Available audio clips. \\
\hline Video & 1- Available videos. \\
\hline search engines & 1- Available. \\
\hline Languages & 1- Available in 27 Languages. \\
\hline Site Customize & 1- Available. \\
\hline $\begin{array}{l}\text { The methods that used in thematic } \\
\text { classification of the media content }\end{array}$ & $\begin{array}{l}\text { 1- Subject classification } \\
\text { 2- A classification of media on the site at the end of } \\
\text { each story highlights related to topics that divided } \\
\text { into four subject headings: places, personalities and } \\
\text { organizations }\end{array}$ \\
\hline $\begin{array}{l}\text { The methods used in the geographical } \\
\text { classification of the media content }\end{array}$ & $\begin{array}{l}\text { 1- Geographic classification within the qualitative and } \\
\text { subject (Middle East, the world). } \\
\text { 2- Geographical division by country }\end{array}$ \\
\hline $\begin{array}{l}\text { Methods of qualitative classification of } \\
\text { media content }\end{array}$ & $\begin{array}{l}\text { 1- Video and Audio, Special Reports, display } \\
\text { Newspaper. }\end{array}$ \\
\hline Interactive Services & $\begin{array}{l}\text { 1- Audience can contribute on the site, BBC Radio and } \\
\text { TV, by sending audio clips, videos, photographs and } \\
\text { uploading them to the site, as well as discussion } \\
\text { forums on the "Share your opinion" pages. } \\
\text { 2- Participating in interactive BBC's television } \\
\text { programs such as the dialogue point, BBC Extra by } \\
\text { answering the questions in the dialogue or send } \\
\text { voice, image, SMS. }\end{array}$ \\
\hline
\end{tabular}

From the above table, it can be observed that the news sites are interested in providing a variety of information materials: texts, audio clips, videos, photo albums and more. In addition to several copies of their sites in languages other than Arabic. The BBC Arabic site is available in other languages, where it provides news, information, services, and technology in 27 languages other than Arabic. The BBC also works to identify places, personalities and organizations through geographical classification within the quality and theme (the Middle East, the world), and geographic division by country.

Interactive services on the BBC News website help attract users and build a trust-based relationship between the site and its audience. Through the interactive services provided on the BBC site, the user is transferred to a sender by allowing the comment or question and email from the site to the recipient as well as sending a text to a third user. The BBC site provides the opportunity for users to use the site, radio, and television and contribute to its development by sending audio clips, video and photographs, and uploading, and participation either by answering questions or dialogue in video and audio or by communication or SMS. 


\section{Study Results and Recommendations}

The results of the research paper reached are the following:

1. The overall appearance of the BBC Arabic site shows that it is committed to the standards of ability to use. The titles are clear and prominent in clear and distinct color and clear text.

2. The site's proficiency results show that the commitment of the $\mathrm{BBC}$ Arabic website to the required standards. The Logo is clearly displayed in the upper right corner of the page, maintaining the same size on all pages.

3. The design of the BBC Arabic site is easy to use because of the logic of organizing the content. There is easy access to information and news on the site. All the paragraphs are on the main page. The browser does not have to enter the internal pages unless viewer want to read articles published in previous days .

4. The site is a portal with several sections of news and reports, weather news, links to social networking sites, and other services.

5. The site also provides abundant information and flexibility in dealing with the text and easy to get what suits the user information directly.

6. Multimedia is one of the most important additions to the website, especially the news sites. The most important multimedia used in news sites is the process of integrating text, graphics, sound, image, links, and tools that allow the user to interact and communicate.

7. $\mathrm{BBC}$ website contain a diversifying the content by providing multimedia that includes text, still images, video, audio, descriptive features of all images, audio and video clips .

8. The BBC Arabic site features a distinctive style in its multimedia display by using playable files with more than one player such as Real Player, Player or Windows Media.

9. The news sites are interested in providing a variety of information materials: texts, audio clips, videos, photo albums and more. In addition to several copies of their sites in languages other than Arabic.

10. The BBC Arabic site is available in other languages, where it provides news, information, services, and technology in 27 languages other than Arabic .

11. The BBC also works to identify places, personalities and organizations through geographical classification within the quality and theme (the Middle East, the world), and geographic division by country.

12. Interactive services on the $\mathrm{BBC}$ News website help attract users and build a trust-based relationship between the site and its audience

The research paper suggests the following:

1. Create the appropriate climate, adequate resources and appropriate cooperation to improve the digital electronic media methods across the Arab world as a primary for Communication with the Public

2. Create the appropriate climate, adequate resources and appropriate cooperation to improve the digital electronic media methods across the Arab world as a primary for Communication with the Public.

\section{References}

Chinwendu , A (2015). The Use of Electronic Media in Nigerian Educational System: Principles, Practice, Problems and Prescriptions. International knowledge sharing platform, 41.

Coroama, V. C., Moberg, Å., \& Hilty, L. M. (2015). Dematerialization through electronic media?. In ICT Innovations for Sustainability (pp. 405-421). Springer, Cham.

Creswell, J. (2012). Educational Research, Planning, Conducting, and evaluating quantitative and qualitative. Fourth Edition. United States of America: Person.

Creswell, J. and Clark, W., (2011) Designing and conducting mixed methods research, 2nd edition. Los Angeles; London: Sage.

David-Ferdon, C., \& Hertz, M. F. (2007). Electronic media, violence, and adolescents: An emerging public health problem. Journal of Adolescent Health, 41(6), S1-S5.

Gurevitch, M., Bennett, T., Curran, J., \& Woollacott, J. (Eds.). (1982). Culture, society and the media (Vol. 
759). London: Methuen.

Handayani, B. (2012). An Examination of Media Convergence and its Implication on Mass Communication Notion. Journal Communication Spectrum, 1(2).

Inglis, A., Joosten, V., \& Ling, P. (2003). Delivering Digitally: Managing the transition to the new knowledge media. Routledge.

Larrondo, A. (2014). News production in the 'post-broadcasting'era: BBC Scotland's move towards convergence. Media, Culture \& Society, 36(7), 935-951.

Malone, T. W., Yates, J., \& Benjamin, R. I. (1994). Electronic markets and electronic hierarchies (pp. 61-83). New York: Oxford University Press.

McQuail, D. (2010). McQuail's mass communication theory. Sage publications.

Meyrowitz, J. (1986). The impact of electronic media on social behavior. Oxford University Press.

Öznacar, B., \& Dagli, G. (2016). The impact of mass media tools on students at the high school level. The Anthropologist, 23(1-2), 152-162.

Quinn, S. (2005). Convergent journalism: The fundamentals of multimedia reporting. Peter Lang.

Roth, W. M. (2010). Reading online news media for science content: A social psychological approach. Reading Psychology, 31(3), 254-281.

Wimmer, R. D., \& Dominick, J. R. (2013). Mass media research. Cengage learning. 